\title{
Erratum to: Potential Effects of Calcium Binding Protein S100A12 on Severity Evaluation and Curative Effect of Severe Acute Pancreatitis
}

\author{
Zhang Feng, ${ }^{1}$ Zhan Yinchu, ${ }^{2,3}$ Shi Yinsheng, ${ }^{2}$ Wu Fengqing, ${ }^{1}$ Zhou Xiaoyang, ${ }^{1}$ Li Jin, ${ }^{1}$ and \\ Gao Xiaofei ${ }^{1}$
}

\section{Erratum to: Inflammation \\ DOI 10.1007/s10753-014-0032-x}

The original version of this article unfortunately contained mistakes. Zhan Yinchu should be captured as the corresponding author and Zhang Feng as the first author. The correct author group now appears above.

Also, the Acknowledgment section was missing in the proof and authors have changes in the affiliation section. Kindly see below:

\section{ACKNOWLEDGMENT}

The study is a project subsided by the fund of science and technology hall of Zhejiang province (2013C33224).

The online version of the original article can be found under at http:// dx.doi.org/10.1007/s10753-014-0032-x.

${ }^{1}$ Zhejiang Chinese Medical University, Hangzhou, 310053, Zhejiang, People's Republic of China

${ }^{2}$ Quzhou People's Hospital, Quzhou, 324000, Zhejiang, People's Republic of China

${ }^{3}$ To whom correspondence should be addressed at Quzhou People's Hospital, Quzhou, 324000, Zhejiang, People's Republic of China. E-mail: jonathan.cheung@foxmail.com 